\title{
AVALIAÇÃo do USO de CARVÃO ATIVADO PREPARADO A PARTIR DE PALHA DO COQUEIRO PARA ADSORÇÃO DE FENOL DE SOLUÇÕES AQUOSAS
}

\author{
M. M. M. SANTOS ${ }^{1}$, J. J. da SILVA ${ }^{2}$, J. H. L. dos $\operatorname{SANTOS}^{3}$ e M. M. M. B. DUARTE ${ }^{4}$ \\ 1,2,3,4 Universidade Federal de Pernambuco, Departamento de Engenharia Química \\ E-mail para contato: maressa_maria16@hotmail.com
}

\begin{abstract}
RESUMO - Os fenóis apresentam efeito nocivo à saúde e ao meio ambiente, sendo necessária sua remoção de efluentes. Neste trabalho foi avaliado o uso de carvão preparado a partir da palha de coqueiro, ativado termicamente com ar sintético, para adsorção de fenol. Foi determinado o ponto de carga zero $\left(\mathrm{pH}_{\mathrm{pcz}}\right)$, avaliado o efeito do $\mathrm{pH}$ inicial da solução e o efeito da concentração de adsorvente (CA). As variáveis CA, granulometria, e velocidade de agitação foram definidas utilizando-se um planejamento fatorial $2^{3}(\mathrm{PF})$. A capacidade adsortiva $q$ foi utilizada para avaliação dos efeitos. $\mathrm{O} \mathrm{pH}_{\mathrm{pcz}}$ para o carvão ativado foi 10 e o processo adsortivo pode ser realizado no $\mathrm{pH}$ 6, natural da solução. A interseção das curvas $q$ e $\%$ de remoção $\left(8\right.$ g. $\left.\mathrm{L}^{-1}\right)$, obtida no estudo da CA, foi adotado como ponto central do PF. O maior $q$ foi obtido para o nível de 4 g. $\mathrm{L}^{-1}, \mathrm{G}<0,090$ mm e $300 \mathrm{rpm}$. Os resultados mostraram que o carvão avaliado é um potencial adsorvente para remoção de fenol, apresentando $q$ de $15 \mathrm{mg} \cdot \mathrm{g}^{-1}$, nas condições estudadas.
\end{abstract}

\section{INTRODUÇÃO}

Dentre os recursos naturais, os recursos hídricos, são os que mais têm sofrido consequências com o descarte impróprio de efluentes industriais contendo compostos fenólicos (Fernandes, 2005). Devido à sua alta toxicidade, esses compostos são classificados como poluentes prioritários (Rodrigues et al., 2011).

Métodos convencionais para tratamento desses efluentes nem sempre são convenientes, completamente eficientes ou ainda apresentam custos elevados (Bhatnagar e Sillanpaa, 2010). Dentre esses processos, os adsortivos destacam-se pela insensibilidade a substâncias tóxicas, simplicidade de seu projeto e operação e facilidades de regeneração. Além de evitar a utilização de solventes tóxicos e minimizar a degradação (Soto et al., 2011).

O carvão ativado é um dos adsorventes mais empregados nos processos adsortivos, principalmente por apresentar grande capacidade de adsorção e diferentes precursores podem ser utilizados em sua fabricação. Os resíduos sólidos apresentam-se como uma boa opção para substituição do carvão ativado comercial (Gonçalves et al., 2007).

Alguns carvões preparados a partir de biomassa vêm sendo avaliados para remoção de 


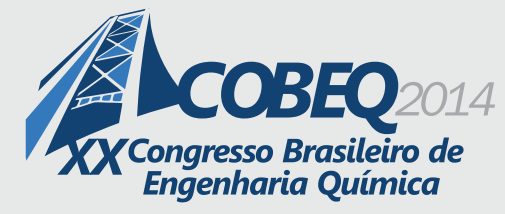

compostos fenólicos, tais como: casca de arroz (Ahmaruzzaman e Sharma, 2005); bagaço de coco in natura (Nogueira et al., 2007); cascas de laranja, borra de café e sementes de melão (Djilani et al., 2012); e serragem de madeira (Larous e Meniai, 2012).

Quando novos adsorventes são avaliados é importante estudar os parâmetros de adsorção, pois o mecanismo de adsorção depende das características químicas e físicas do material adsorvente, do adsorbato e das condições experimentais (Vieira et al., 2009).

Este trabalho teve por objetivo avaliar o uso de carvão ativado termicamente com ar sintético, preparado a partir da palha de coqueiro, um resíduo agroindustrial, para adsorção de fenol em solução aquosa.

\section{METODOLOGIA}

O carvão da palha de coqueiro foi ativado em forno elétrico (Lindberg, Blue M) onde as amostras foram colocadas em um reator cilíndrico de quartzo conectado a fluxos de gases. O aquecimento foi realizado partindo-se da temperatura ambiente até $873 \mathrm{~K}$, sob fluxo de nitrogênio à vazão de $100 \mathrm{~mL} \cdot \mathrm{min}^{-1}$, permanecendo nesta condição por 1 hora. A ativação ocorreu pela troca do fluxo por ar sintético à vazão de $100 \mathrm{~mL} \cdot \mathrm{min}^{-1}$ durante 1 hora, utilizando o mesmo sistema.

As soluções de fenol foram preparadas a partir de fenol cristal p.a. (Ácido fênico, peso molecular 94,11 g.mol ${ }^{-1}$, marca Dinâmica).

Os experimentos de adsorção foram realizados em banho finito, utilizando uma mesa agitadora (Ika, KS 130 control), à temperatura ambiente do laboratório $\left(25^{\circ} \mathrm{C} \pm 2^{\circ} \mathrm{C}\right)$. Ensaios em branco foram realizados seguindo-se o mesmo procedimento das amostras.

Ao final de cada ensaio as amostras foram filtradas e os teores do fenol foram quantificados antes e após a execução dos experimentos de sorção através de Espectrômetro UV-Visível (Thermo Scientific, Genesys 10S), no comprimento de onda de $270 \mathrm{~nm}$. O limite de detecção do método foi de $0,09 \mathrm{mg} \cdot \mathrm{L}^{-1}$, limite de quantificação de $0,3 \mathrm{mg} \cdot \mathrm{L}^{-1}$, faixa linear de trabalho de 0,5 a $100 \mathrm{mg} \cdot \mathrm{L}^{-1}$.

A quantidade fenol adsorvida por massa de adsorvente (capacidade adsortiva, $q$ ) foi calculada utilizando a Equação 1:

$$
q=\frac{\left(C_{o}-C_{f}\right) V}{M}
$$

sendo: $q_{e}$ a quantidade de fenol adsorvida dado em miligrama de adsorvato por grama de adsorvente $\left(\mathrm{mg} . \mathrm{g}^{-1}\right) ; C_{0}$ a concentração inicial de fenol $\left(\mathrm{mg} . \mathrm{L}^{-1}\right) ; C_{f}$ a concentração final de fenol $\left(\mathrm{mg} . \mathrm{L}^{-1}\right) ; \quad V$ o volume da solução em litros e $m$ a massa de adsorvente em gramas.

$\mathrm{O} \mathrm{pH}$ do ponto de carga zero $\left(\mathrm{pH}_{\mathrm{pcz}}\right)$ do adsorvente foi estimado por medidas de $\mathrm{pH}$ da água antes e após o contato com o sólido. Foram adicionados $0,25 \mathrm{~g}$ do adsorvente em $25 \mathrm{~mL}$ da água com 


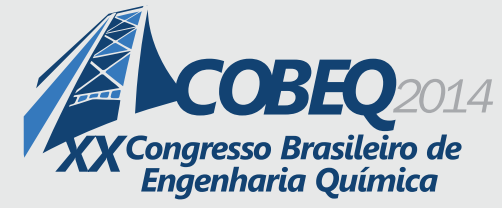

pH variando de 2 a 11, ajustado com soluções de ácido clorídrico $\left(0,1 \mathrm{~mol} . \mathrm{L}^{-1}\right)$ e hidróxido de sódio $\left(0,1 \mathrm{~mol} . \mathrm{L}^{-1}\right)$ e as soluções ficaram sob agitação de $300 \mathrm{rpm}$ por 24 horas. $\mathrm{O} \mathrm{pH}_{\mathrm{pcz}}$ foi obtido pelo gráfico $\left(\mathrm{pH}_{\text {final }}-\mathrm{pH}_{\text {inicial }}\right)$ vs. $\mathrm{pH}_{\text {inicial }}$, na interseção da curva com o eixo $\mathrm{pH}_{\text {inicial. }}$.

O efeito do pH inicial da solução fenólica $\left(100 \mathrm{mg} \cdot \mathrm{L}^{-1}\right)$ foi investigado nos níveis de pH 2 a 10 . Foram adicionados $0,25 \mathrm{~g}$ do adsorvente a $25 \mathrm{~mL}$ das soluções nos respectivos $\mathrm{pH}$ e ficaram sob agitação de 300 rpm por 6 horas.

O efeito da concentração do adsorvente foi avaliado na faixa de 4 a 40 g. $\mathrm{L}^{-1}$ e os ensaios foram realizados utilizando $25 \mathrm{~mL}$ da solução fenólica $\left(100 \mathrm{mg} . \mathrm{L}^{-1}\right)$ sob agitação de $300 \mathrm{rpm}$ por 6 horas.

De forma a se obter uma maior eficiência nos processos adsortivos foi realizado um planejamento fatorial para avaliar as influências das variáveis sobre os processos e determinar as melhores condições de trabalho.

Os ensaios foram realizados em ordem aleatória e o ponto central em triplicata. A inclusão do ponto central tem como objetivo permitir teste de falta de ajuste do modelo empírico a ser proposto. Para os processos adsortivos foram avaliadas as variáveis: Concentração do adsorvente - C.A. (4, 8 e 12 g. $\left.\mathrm{L}^{-1}\right)$, granulometria do adsorvente $-\mathrm{G}(<0,090 \mathrm{~mm}, 0,090-0,150 \mathrm{~mm}$ e $0,150-0,212 \mathrm{~mm})$ e velocidade de agitação - V.A. (0; 150 e 300 rpm).

A resposta utilizada para determinar a eficiência do processo foi a capacidade de adsorção ( $q$ em mg. $\left.\mathrm{g}^{-1}\right)$. Os cálculos dos efeitos dos fatores e as interações entre eles com os seus respectivos erros padrão foram realizados segundo Barros Neto et al. (2007), com auxilio do software Statistica for Windows 6.0.

Os ensaios do planejamento fatorial foram realizados utilizando erlenmeyers contendo $25 \mathrm{~mL}$ da solução fenólica na concentração de $100 \mathrm{mg} \cdot \mathrm{L}^{-1}$, por 6 horas.

\section{RESULTADOS E DISCUSSÃO}

\subsection{Ponto de Carga Zero}

Chama-se de Ponto de carga zero $\left(\mathrm{pH}_{\mathrm{pcz}}\right)$ o valor de $\mathrm{pH}$ em que a carga superficial do carvão é zero. O gráfico interceptou o eixo horizontal $\left(\mathrm{pH}_{\text {inicial }}\right)$ em 10 , conforme a Figura 1 , que corresponde ao $\mathrm{pH}_{\mathrm{pcz}}$ do adsorvente. Em $\mathrm{pH}$ inferiores a 10 o carvão ativado termicamente com ar sintético, preparado a partir da palha de coqueiro, possui carga superficial positiva favorecendo a adsorção de ânions, como o fenol.

Rodrigues et al. (2011) encontraram $\mathrm{pH}_{\mathrm{pcz}}$ no valor de 9,7 para carvão obtido do caroço de abacate ativado por fluxo de $\mathrm{CO}_{2}$. 


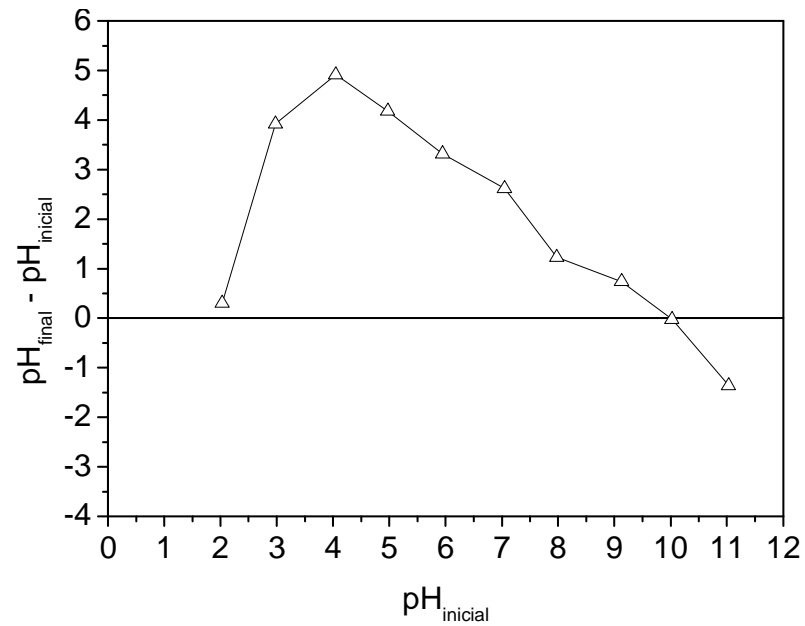

Figura 1 - Ponto de carga zero. pH 2 a 11, V.A. $=300$ rpm, C.A. $=4$ g. $\mathrm{L}^{-1}, \mathrm{t}=24 \mathrm{~h}$.

Fernandes (2005) definiu os carvões ativados com vapor d'água (carvão ativado da pinha e carvão ativado da serragem) como alcalinos, por apresentarem $\mathrm{pH}_{\mathrm{pcz}}$ superior ao $\mathrm{pH} 7$.

\subsection{Influência do pH Inicial da Solução}

A capacidade adsortiva do carvão ativado, com relação a diferentes valores de $\mathrm{pH}$ inicial da solução fenólica, é mostrada pela Figura 2.

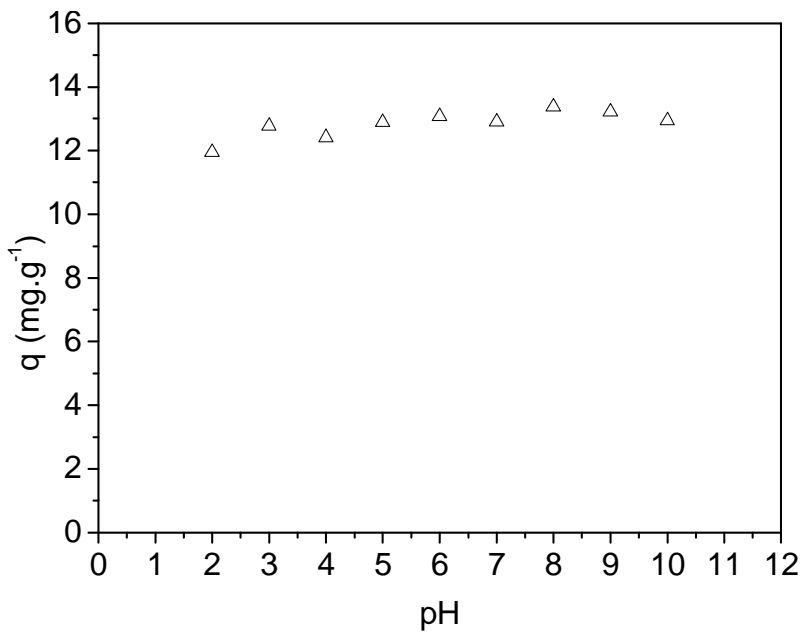

Figura 2 - Influência do pH inicial da solução fenólica. pH 2 a 10, V.A. $=300$ rpm, t $=6$ h.

Observa-se na Figura 2 que não há variações significativas em relação à adsorção do fenol para os diferentes valores de $\mathrm{pH}$, isto porque os valores estudados encontram-se abaixo do $\mathrm{pH}_{\mathrm{pcz}}$. Os experimentos de adsorção foram realizados no $\mathrm{pH}$ natural da solução fenólica, $\mathrm{pH}$ 6. Segundo Rodrigues et al. (2011), carvões ativados apresentam uma ampla faixa predominantemente positiva, isto pode ser constatado no estudo do ponto de carga zero (Figura 1). 


\subsection{Influência da concentração do adsorvente}

O efeito da variação da concentração do adsorvente na adsorção de fenol é apresentado na Figura 3. Como pode ser observada a remoção do fenol aumentou significativamente com a elevação da concentração do adsorvente chegando a uma remoção de aproximadamente 100\% em 6 horas.

Entretanto, a quantidade do fenol adsorvida por unidade de massa do adsorvente diminuiu com o aumento da concentração do adsorvente, tendo em vista a redução da razão adsorvato/adsorvente. A capacidade adsorvida diminuiu de $12,72 \mathrm{mg} \mathrm{g}^{-1}$ (49,6\% de remoção) para $2,53 \mathrm{mg} \cdot \mathrm{g}^{-1}$ (98,7\% de remoção).

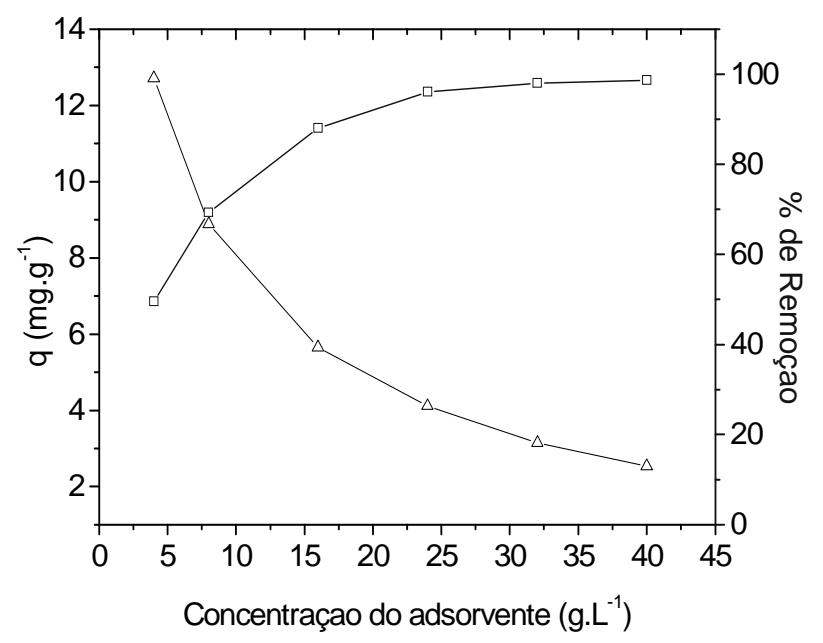

Figura 3 - Influência da concentração. C.A. de 4 a 40 g.L $\mathrm{L}^{-1}, \mathrm{C}_{0}=100$ mg.L $\mathrm{L}^{-1}$, V.A. $=300$ rpm, $\mathrm{t}=6 \mathrm{~h}, \mathrm{pH}=6$.

A concentração do adsorvente de 8 g.L $\mathrm{L}^{-1}$ correspondente a interseção entre as curvas foi utilizada como ponto central para o planejamento fatorial visando encontrar uma melhor relação entre $\%$ de remoção e capacidade adsortiva.

\subsection{Definição das Condições de Trabalho}

Os efeitos principais e de interação foram estatisticamente significativos para um nível de $95 \%$ de confiança nos níveis estudados (valores maiores que $\mathrm{p}=0,05$ ), o que pode ser constatado através da carta de Pareto apresentada na Figura 4. 


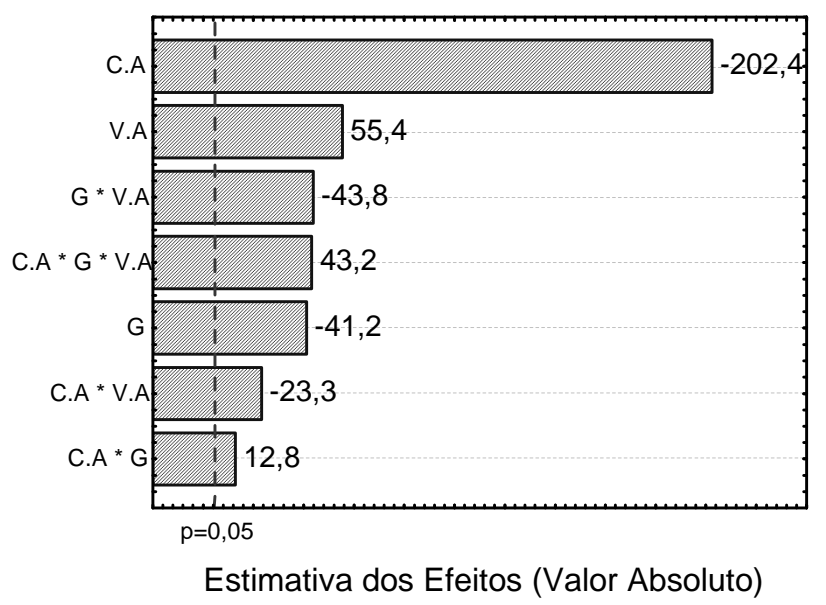

Figura 4 - Carta de Pareto dos efeitos calculados para o carvão ativado (erro puro de 0,002).

As superfícies de resposta referentes às interações de dois fatores estatisticamente significativos estão apresentadas na Figura 5. A maior capacidade adsortiva $\left(15 \mathrm{mg} \cdot \mathrm{g}^{-1}\right)$ foi obtida para o nível 4 g. $\mathrm{L}^{-1}$ do adsorvente, granulometria de $<0,090 \mathrm{~mm}$ e $300 \mathrm{rpm}$.
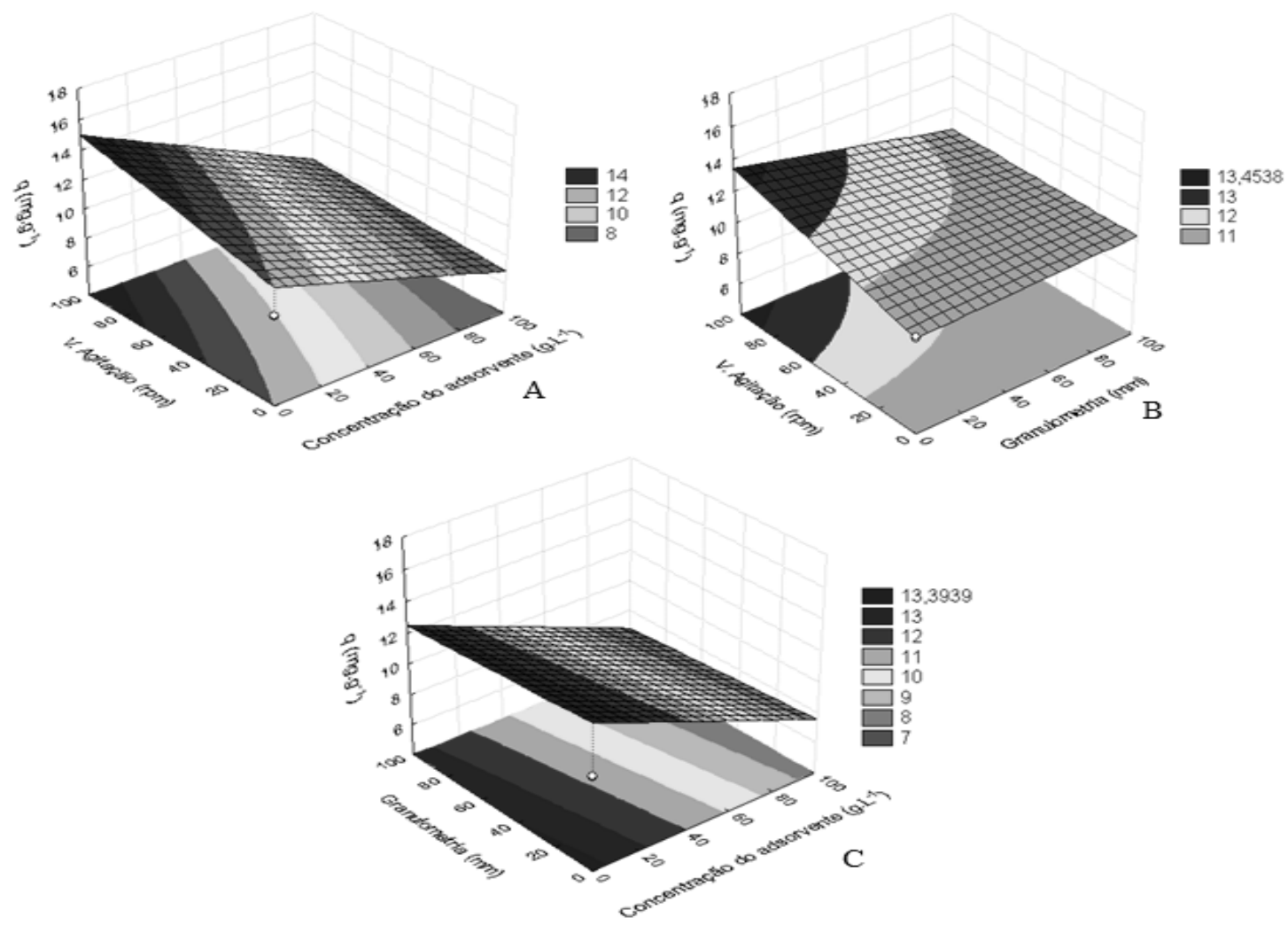

Figura 5 - Superfícies de resposta para a capacidade adsortiva, usando carvão ativado obtido da palha do coqueiro como adsorvente. (A) C.A vs. V.A.; (B) G. vs. V.A. e (C) C.A vs. G. 
A partir dos dados experimentais e utilizando o programa Statistica for Windows 6.0 foi obtido um modelo empírico capaz de prever a capacidade adsortiva $q$ do carvão ativado para o fenol, dentro do intervalo de estudo, para as três variáveis (Equação 2).

$q\left(\mathrm{mg} . \mathrm{g}^{-1}\right)=10,27-3,05$ C.A. $-0,62 \mathrm{G}+0,83$ V.A. $+0,19$ C.A $\times \mathrm{G}-0,35$ C.A. $\times$ V.A $-0,66$ G x V.A. $+0,65$ C.A. $\times$ V.A $\times$ G Figura 6.

O gráfico de valores previstos no modelo empírico e dos valores observados é apresentado na

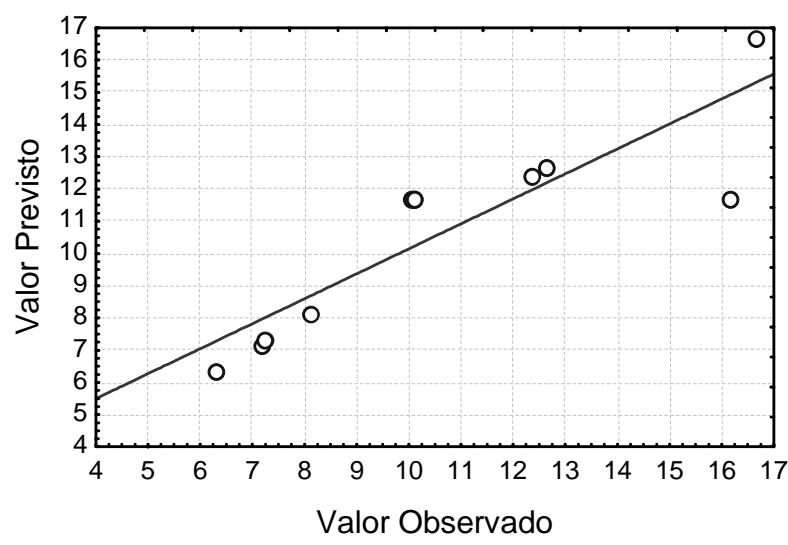

Figura 6 - Gráfico dos valores previstos no modelo empírico versus valores observados experimentalmente.

Através da Figura 6, observa-se que as previsões do modelo empírico se adequam aos resultados observados.

\section{CONCLUSÃO}

Os valores da capacidade adsortiva foram próximos na faixa de $\mathrm{pH}$ estudada, este resultado corrobora com o obtido para $\mathrm{o} \mathrm{pH}_{\mathrm{pcz}}$, pois o carvão possui ampla faixa positiva favorecendo a adsorção do fenol. A concentração de adsorvente 8 g. $\mathrm{L}^{-1}$ foi utilizada como ponto central para o planejamento fatorial, pois neste ponto houve a interseção das curvas, indicando a existência de uma boa relação entre o percentual de remoção e a capacidade adsortiva do carvão ativado.

A maior capacidade adsortiva foi obtida para 4 g. $\mathrm{L}^{-1}$ do adsorvente, granulometria de $<0,090$ mm e $300 \mathrm{rpm}$, para os níveis estudados. O carvão ativado preparado a partir da palha do coqueiro, avaliado neste trabalho, apresentou-se como uma alternativa técnica viável para o tratamento de efluentes contendo fenol.

\section{AGRADECIMENTOS}

A Elephant Indústria Química Ltda. por fornecer o carvão utilizado no trabalho e ao PRH28/ANP e Petrobras pelo apoio e bolsa IC concedida 


\section{REFERÊNCIAS}

AHMARUZZAMAN, M.; SHARMA, D.K. Adsorption of phenols from wastewater. J. Colloid Interface Sci., v. 287, p. 14-24, 2005.

BARROS NETO, B.; SCARMINIO, I. S.; BRUNS, R. E. in Como Fazer Experimentos: Pesquisa e desenvolvimento na ciência e na indústria, $3^{\text {a }}$ Ed.; Unicamp, Campinas-SP, 2007.

BHATNAGAR, A.; SILLANPÄÄ, M. Utilization of agro-industrial and municipal waste materials as potential adsorbents for water treatment - A review. Chem. Eng. J., v. 157, p. 277296, 2010.

DJILANI C.; ZAGHDOUDIB R.; MODARRESSID A.; ROGALSKID M.; DJAZIA F.; LALLAME A.. Elimination of organic micropollutants by adsorption on activated carbono prepared from agricultural waste. Chem. Eng. J., v. 189-190, p. 203-212, 2012.

FERNANDES, R. Adsorventes alternativos para remoção de fenol em solução aquosa. Dissertação de Mestrado. Florianópolis, SC: UFSC, 2005.

GONÇALVES M.; GUERREIRO M. G.; BIANCHI M. L.; OLIVEIRA L. C. A.; PEREIRA E. I.; DALLAGO R. M. Produção de carvão a partir de resíduo de erva-mate para a remoção de contaminantes orgânicos de meio aquoso. Ciênc. Agrotec., v. 31, n. 5, p. 1386-1391, 2007.

LAROUS S.; MENIAI A.H. The use of sawdust as by product adsorbent of organic pollutant from wastewater: adsorption of phenol. Energy Procedia, v. 18, p. 905-914, 2012.

NOGUEIRA, Ma I. C.; NASCIMENTO, R. F.; AGUIAR, S. O. ; BUARQUE, H. L. B.; ARAÚJO, R. S. Adsorção de fenol e nitrofenóis em solução aquosa usando bagaço da casca de coco como adsorvente. In: $2^{\circ}$ Congresso de Pesquisa e Inovação da Rede Norte Nordeste de Educação Tecnológica, João Pessoa, 2007, 80-84.

RODRIGUES, L.A.; SILVA, M.C.P.; ALVAREZ-MENDES, M.O.; COUTINHO, A.R.; THIM, G.P. Phenol removal from aqueous solution by activated carbon produced from avocado kernel seeds. Chem. Eng. J., v. 174, p. 49- 57, 2011.

SOTO M.L.; MOURE A.; DOMINGUEZ H.; PARAJÓ J.C. Recovery, concentration and purification of phenolic compounds by adsorption- A review. J. Food Eng., v.105, p.1-27, 2011.

VIEIRA, A.P.; SANTANA, S.A.A.; BEZERRA, C.W.B.; SILVA, H.A.S.; CHAVES, J.A.P.; MELO, J.C.P.; SILVA FILHO, E.C.; AIROLDI, C. Kinetics and thermodynamics of textile dye adsorption from aqueous solutions using babassu coconut mesocarpo. J. Hazard. Mater., v. 166, p. 1272-1278, 2009. 